\title{
Food insecurity in veteran households: findings from nationally representative data
}

\author{
Daniel P Miller ${ }^{1, *}$, Mary Jo Larson ${ }^{2}$, Thomas Byrne ${ }^{1}$ and Ellen DeVoe ${ }^{1}$ \\ ${ }^{1}$ Boston University School of Social Work, 264 Bay State Road, Boston, MA 02215, USA: ${ }^{2}$ The Heller School for \\ Social Policy and Management, Institute for Behavioral Health, Waltham, MA, USA
}

Submitted 11 June 2015: Final revision received 11 August 2015: Accepted 24 September 2015: First published online 10 November 2015

\begin{abstract}
Objective: The present study is the first to use nationally representative data to compare rates of food insecurity among households with veterans of the US Armed Forces and non-veteran households.

Design: We used data from the 2005-2013 waves of the Current Population Survey - Food Security Supplement to identify rates of food insecurity and very low food security in veteran and non-veteran households. We estimated the odds and probability of food insecurity in veteran and non-veteran households in uncontrolled and controlled models. We replicated these results after separating veteran households by their most recent period of service. We weighted models to create nationally representative estimates.

Setting: Nationally representative data from the 2005-2013 waves of the Current Population Survey - Food Security Supplement.

Subjects: US households ( $n 388$ 680).

Results: Uncontrolled models found much lower rates of food insecurity $(8.4 \%)$ and very low food security $(3.3 \%)$ among veteran households than in non-veteran households ( $14.4 \%$ and $5.4 \%$, respectively), with particularly low rates among households with older veterans. After adjustment, average rates of food insecurity and very low food security were not significantly different for veteran households. However, the probability of food insecurity was significantly higher among some recent veterans and significantly lower for those who served during the Vietnam War.

Conclusions: Although adjusting eliminated many differences between veteran and non-veteran households, veterans who served from 1975 and onwards may be at higher risk for food insecurity and should be the recipients of targeted outreach to improve nutritional outcomes.
\end{abstract}

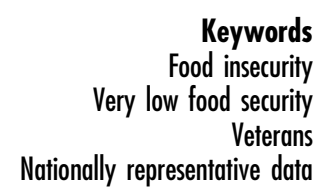

Food insecurity

terans

Nationally representative data
Recent findings point to high levels of food insecurity (FI) in veteran households. A 2012 survey of Minnesota-based veterans using the US Department of Veteran Affairs health-care system found high rates (nearly $27 \%$ ) of FI among veterans of the wars in Iraq and Afghanistan ${ }^{(1)}$ and an analysis of data on veterans from the Veterans Aging Cohort Study who were also engaged with the US Department of Veteran Affairs found that nearly a quarter expressed some concern about having adequate food for themselves or their families ${ }^{(2)}$. The Feeding America organization also gained national attention when it estimated that one in four active duty or reserve military households had sought food assistance from its national network of emergency food providers ${ }^{(3)}$. Data from the same report indicated that $15.3 \%$ of emergency food client households had at least one member who had ever served in the US military ${ }^{(4)}$.
Although these reports speak to the potential for high levels of food need and FI in US veteran households, they were limited by their reliance on highly selected samples $^{(1-3)}$, lack of a comparison group of non-veteran households $^{(1,2)}$ or limited measures for $\mathrm{FI}^{(2)}$. FI, which indicates that household 'access to adequate food [is] limited by a lack of money or other resources, ${ }^{(5)}$, is an important indicator of health for both children and adults $^{(6-8)}$. Thus, documenting FI rates among veteran households is an important public health surveillance goal. Higher rates of veteran FI in national data would provide an important call to action for state and federal government to improve supports for veterans and their families.

Indeed, there is reason to believe that veteran households may be more likely to be food insecure than the average US household. FI is a measure of economic hardship. And while income is strongly and negatively 
associated with household $\mathrm{FI}^{(5,9)}$, it is not the sole determinant and other factors relevant to management of household resources are also important. For example, research points to a connection between household FI and adult psychological well-being ${ }^{(10)}$, including psychosocial problems like post-traumatic stress disorder, depression, binge drinking and substance use disorders that may be associated with military service ${ }^{(11-19)}$.

The transition from military to civilian life often presents unique barriers to financial security, which may amplify the risk of experiencing FI. New veterans may experience challenges in the labour market due to a mismatch between skills acquired during military service and those required for civilian employment ${ }^{(20,21)}$. In addition, the provision of basic needs as a by-product of military life may obviate the need for service members to develop sound money management skills ${ }^{(22,23)}$, which could translate into an increased risk for FI and other forms of economic hardship in civilian life.

On the other hand, veteran households may have important advantages that promote household food security. For one, the Veterans Health Administration provides $29 \%$ of male veterans and $25 \%$ of female veterans with regular and comprehensive medical care ${ }^{(24)}$, which accounts for higher rates of health insurance coverage among veterans relative to civilians ${ }^{(25)}$. In addition, the Veterans Benefits Administration administers programmes that offer educational assistance, vocational training, employment services, specialty home loans and income support to certain groups of low-income veterans ${ }^{(26)}$. Veterans facing housing crises may be eligible for a range of specialized programmes ${ }^{(27)}$. Access to this array of benefits may decrease the risk of FI among veterans relative to their non-veteran peers, although evidence suggests that veteran households are less likely to participate in the Supplemental Nutrition Assistance Program (SNAP) ${ }^{(28)}$, indicating that veteran status is not a guarantee of access to benefits.

Veterans' military experiences and household variables are diverse and may be important to consider. Length of service, combat exposure and role vary among veterans both within and across periods of service, and service era itself may demarcate important differences that could affect the risk of FI. Specifically, those who enlisted after the shift to an all-volunteer force rank less favourably than their non-veteran peers in terms of socio-economic status, educational attainment ${ }^{(29)}$ and the presence of behavioural health problems ${ }^{(29-32)}$, all of which are hypothesized to account for the increased risk of adverse social $^{(33,34)}$ and economic ${ }^{(35)}$ outcomes.

Two previous studies of material hardship examined whether household food insufficiency (a more limited measure of food hardship available in the Survey of Income and Program Participation) varied by the presence of veteran and disability status ${ }^{(36,37)}$. In comparison to households with no veterans and no disabled residents, the first study found that the odds of food insufficiency were significantly higher among disabled veteran households and the second found higher odds of food insufficiency among older adult households with disabled veterans and those with non-disabled veterans ${ }^{(36,37)}$. However, these two studies were limited by a focus on food insufficiency only, which is much rarer than FI and based only on a single survey item. Indeed, in the second study, no non-disabled veteran households reported food insufficiency, making comparisons with this group impossible ${ }^{(36)}$.

This review of the literature points to the possibility for both higher and lower rates of FI in veteran homes. Further, it suggests the importance of accounting for differences between veteran and non-veteran households and for distinguishing among different veteran cohorts. Using nationally representative data from 2005-2013 waves of the Current Population Survey - Food Security Supplement (CPS-FSS), the present study was guided by two specific aims:

1. to provide reliable and contemporary national estimates of household FI and very low food security (VLFS) by veteran status and most recent period of military service; and

2. to identify whether there are significant differences in rates of FI and VLFS after controlling for differences between veteran and non-veteran households.

\section{Methods}

\section{Data}

We pooled data from the 2005-2013 waves of the CPS-FSS ${ }^{(38)}$. The CPS-FSS is administered annually as a supplement to the December Current Population Survey (CPS) and provides national estimates of FI, which are published by the US Department of Agriculture ${ }^{(5,38,39)}$. Respondents to the CPS-FSS first complete the regular CPS which includes information on labour force participation, household demographics and composition along with questions related to current and previous military service. The CPS-FSS asks about FI, participation in food and nutrition programmes, and other household food dynamics. In each year, a portion of CPS households elected not to participate in the CPS-FSS. Thus, all of our analyses rely upon household-level supplement weights, which account for non-response and make the sample representative of the non-institutionalized population of the USA. About $0.9 \%$ of cases were missing data on one or more variables and were dropped from the present analysis. Our final analytic sample consisted of 388680 households from the nine survey years. Because all analyses were conducted with de-identified secondary data with no means to link information to individual respondents, the present study was considered to be not human subjects research and required no review by an institutional review board. 


\section{Measures}

\section{Food insecurity}

The CPS-FSS contains the eighteen-item Food Security Module developed by the Economic Research Service of the US Department of Agriculture. The Module contains ten adult-referenced questions and eight child-referenced questions asked only of those households with children ${ }^{(40)}$ (see online supplementary material, Supplemental Table 1). We used constructed variables in the CPS-FSS ${ }^{(40,41)}$ to classify households as having been food secure or having experienced FI or VLFS over the previous 12 months. VLFS is a particularly severe form of hardship and occurs when households limit food intake or experience disrupted eating patterns because of limited money or other resources ${ }^{(5)}$.

\section{Veteran status and period of service}

The monthly CPS asked all respondents aged 17 years and older 'Did you ever serve on active duty in the US Armed Forces?' We coded respondents as veterans if they answered yes but were not currently in the Armed Forces and identified veteran households as those with at least one resident veteran. The CPS also asked veterans to identify up to four periods of service, with the following choices: 'September 2001 or later', 'August 1990 to August 2001', 'May 1975 to July 1990', 'Vietnam War (August 1964 to April 1975)', 'February 1955 to July 1964', 'Korean War (July 1950 to January 1955)', 'January 1947 to June 1950', 'World War II (December 1941 to December 1946)' and 'November 1941 or earlier'. Because of small sample sizes, we recoded the last four of these groups into a broader category of 'Korean War or earlier'. We coded each veteran's most recent period of service and, in households with multiple veterans $(0.7 \%$ of the sample and $3.5 \%$ of all veteran households), used the service of the veteran who had most recently been in the Armed Forces as the measure for the entire household.

\section{Control variables}

A primary aim of our analyses was to investigate whether differences in FI between veteran and non-veteran households persisted after controlling for other factors that might otherwise account for these differences. As noted above, veteran households might have previous or ongoing access to a number of programmes or benefits that could also be related to their food security status, including education, job training and housing programmes, or regular medical care.

Because we were unable to examine directly which benefits veterans received, our analyses controlled for a number of factors that might index their ultimate effect: housing tenure (housing was 'owned or being purchased', 'rented for cash' or 'occupied without payment') and housing type ('house/apartment/flat', 'hotel or motel', 'mobile home' or 'other'); highest level of household education ('less than high school', 'high school', 'some college', 'bachelor's degree' or 'graduate school or higher'); current labour force status ('employed', 'unemployed', 'out of the labour force', 'disabled' or 'active military' (in non-veteran households only)); and receipt of food and nutrition assistance ('SNAP/Food Stamp participation', 'WIC (Special Supplemental Nutrition Program for Women, Infants, and Children) participation' or receipt of 'free or reduced-price meals' from the National School Lunch Program, School Breakfast Program or a Head Start programme (coded as 0 for households without children)). To account for the potential accumulation of such benefits, we included an additional control for the presence of multiple veterans in the home.

In addition, we controlled for a number of sociodemographic factors, some of which also might act as confounders: respondent sex; marital status ('married, spouse present', 'married, spouse absent', 'widowed', 'divorced', 'separated' or 'never married'); race/ethnicity ('white not Hispanic', 'black not Hispanic', 'Hispanic any race', 'American Indian not Hispanic', 'Asian/Pacific Islander not Hispanic' or 'multiple race, not Hispanic'); household poverty ('income above $185 \%$ of the federal poverty line', 'income below $185 \%$ of the federal poverty line' or 'income not reported' (only in 2009 or earlier)); the presence of immigrants in the household; the number of children and number of adults in the household; and age and age-squared (to account for the curvilinear relationship between age and household FI). Lastly, to rule out secular effects and potential unobserved differences in state policies or economic climate that could benefit (or detriment) veteran households, we included indicators for state of residence and survey year. We coded personlevel variables (e.g. marital status, race/ethnicity, age, sex) based on data from the survey respondent in non-veteran households, from the veteran in households with only one veteran resident and from the veteran with the most recent period of service in multiple-veteran homes.

\section{Analyses}

Using the statistical software package Stata version 13, we ran two sets of analyses. First, we specified uncontrolled and controlled logistic regression models comparing rates of FI and VLFS in veteran and non-veteran households. Next, we re-ran these analyses after separating veteran households into most recent period of service. To ease interpretation of our regression results, we generated predicted probabilities of FI and VLFS according to veteran status and most recent period of military service. In supplemental analyses (available upon request), we re-specified all models first using probit regression, next after clustering standard errors at the state level and finally by dropping multiple-veteran households. In addition, because CPS households are surveyed for four months, drop out of the survey for eight months and then are surveyed again for another four months, we ran supplemental models clustering standard errors by household identification number to account for repeat households. Results from all of these models were nearly identical to those shown below. 


\section{Results}

As shown in Table 1, just over $17 \%$ of the sample was comprised of veteran households. The group with most recent service in the Vietnam War was the largest group of veterans (over $30 \%$ of all veteran households). More than
$13 \%$ of all households experienced FI in the past 12 months and $5 \cdot 1 \%$ experienced VLFS.

Table 2 presents the results of uncontrolled and controlled logistic regressions. In the unadjusted models, veteran households had significantly lower odds of FI $(\mathrm{OR}=0.547, P<0.001)$ and the probability of FI was 0.084

Table 1 Descriptive statistics of the study sample: US households ( $n 388$ 680) from 2005-2013 waves of the Current Population Survey - Food Security Supplement†

\begin{tabular}{|c|c|c|}
\hline & $\begin{array}{l}\text { Proportion or mean/sD } \\
\text { (for continuous variables) }\end{array}$ & Range \\
\hline Veteran household & 0.171 & $0-1$ \\
\hline \multicolumn{3}{|l|}{ Most recent period of service } \\
\hline September 2001 or later & 0.013 & $0-1$ \\
\hline August 1990 to August 2001 & 0.020 & $0-1$ \\
\hline May 1975 to July 1990 & 0.032 & $0-1$ \\
\hline Vietnam War (August 1964 to April 1975) & 0.052 & $0-1$ \\
\hline February 1955 to July 1964 & 0.022 & $0-1$ \\
\hline Korean War or earlier & 0.033 & $0-1$ \\
\hline Household food insecurity & 0.134 & $0-1$ \\
\hline Household very low food security & 0.051 & $0-1$ \\
\hline Multiple-veteran household & 0.006 & $0-1$ \\
\hline \multicolumn{3}{|l|}{ Labour force status $\ddagger$} \\
\hline Employed & 0.620 & $0-1$ \\
\hline Unemployed & 0.043 & $0-1$ \\
\hline Out of the labour force & 0.275 & $0-1$ \\
\hline Disabled & 0.058 & $0-1$ \\
\hline Active military (non-veterans only) & 0.003 & $0-1$ \\
\hline Female & 0.452 & $0-1$ \\
\hline Age (years) $\ddagger$, mean & 49.94 & $15-85$ \\
\hline SD & 0.032 & \\
\hline \multicolumn{3}{|l|}{ Race/ethnicity $\ddagger$} \\
\hline White, not Hispanic & 0.702 & $0-1$ \\
\hline Black, not Hispanic & 0.122 & $0-1$ \\
\hline Hispanic, any race & 0.117 & $0-1$ \\
\hline American Indian, not Hispanic & 0.006 & $0-1$ \\
\hline Asian/Pacific Islander, not Hispanic & 0.041 & $0-1$ \\
\hline Multiple race, not Hispanic & 0.011 & $0-1$ \\
\hline \multicolumn{3}{|l|}{ Martial statusł } \\
\hline Married, spouse present & 0.494 & $0-1$ \\
\hline Married, spouse absent & 0.017 & $0-1$ \\
\hline Widowed & 0.098 & $0-1$ \\
\hline Divorced & 0.150 & $0-1$ \\
\hline Separated & 0.030 & $0-1$ \\
\hline Never married & 0.210 & $0-1$ \\
\hline \multicolumn{3}{|l|}{ Maximum household education } \\
\hline Less than high school & 0.077 & $0-1$ \\
\hline High school & 0.245 & $0-1$ \\
\hline Some college & 0.300 & $0-1$ \\
\hline Bachelor's degree & 0.227 & $0-1$ \\
\hline Graduate school or higher & 0.151 & $0-1$ \\
\hline Number of children, mean & 0.621 & $0-12$ \\
\hline SD & 0.002 & \\
\hline Number of adults, mean & 1.885 & $0-12$ \\
\hline SD & 0.002 & \\
\hline \multicolumn{3}{|l|}{ Housing type } \\
\hline House/apartment/flat & 0.950 & $0-1$ \\
\hline Hotel or motel & 0.001 & $0-1$ \\
\hline Mobile home & 0.047 & $0-1$ \\
\hline Other & 0.001 & $0-1$ \\
\hline \multicolumn{3}{|l|}{ Housing tenure } \\
\hline Owned or being purchased & 0.674 & $0-1$ \\
\hline Rented for cash & 0.313 & $0-1$ \\
\hline Occupied without payment & 0.013 & $0-1$ \\
\hline Immigrant household & 0.165 & $0-1$ \\
\hline \multicolumn{3}{|l|}{ Household in poverty } \\
\hline No & 0.608 & $0-1$ \\
\hline Yes & 0.288 & $0-1$ \\
\hline Not reported (2009 and earlier) & 0.104 & $0-1$ \\
\hline \multicolumn{3}{|l|}{ Receipt of food and nutrition assistance } \\
\hline SNAP/Food Stamps participation & 0.086 & $0-1$ \\
\hline WIC participation & 0.028 & $0-1$ \\
\hline Free or reduced-price meals participation & 0.072 & $0-1$ \\
\hline
\end{tabular}

SNAP, Supplemental Nutrition Assistance Program; WIC, Special Supplemental Nutrition Program for Women, Infants, and Children. †All estimates are based on the use of sample weights provided by the Current Population Survey.

$\ddagger$ Variable applies to the head of household in non-veteran homes or the veteran with the most recent service in veteran homes. 
Table 2 Unadjusted and adjusted past-year household food insecurity and very low food security by veteran status among US households ( $n 388$ 680) from 2005-2013 waves of the Current Population Survey - Food Security Supplement†

\begin{tabular}{|c|c|c|c|c|c|c|}
\hline & \multicolumn{3}{|c|}{ Food insecurity } & \multicolumn{3}{|c|}{ Very low food security } \\
\hline & Probability & OR & $95 \% \mathrm{Cl}$ & Probability & OR & $95 \% \mathrm{Cl}$ \\
\hline \multicolumn{7}{|l|}{ Unadjusted results } \\
\hline \multicolumn{7}{|l|}{ Veteran household } \\
\hline Noł & 0.144 & & & 0.054 & & \\
\hline Yes & 0.084 & $0.547^{\star \star \star}$ & $0.529,0.566$ & 0.033 & $0.601^{\star \star \star}$ & $0.570,0.633$ \\
\hline \multicolumn{7}{|l|}{ Adjusted results } \\
\hline \multicolumn{7}{|l|}{ Veteran household } \\
\hline Noł & 0.133 & & & 0.051 & & \\
\hline Yes & 0.135 & 1.014 & $0.972,1.057$ & 0.051 & 1.008 & $0.947,1.072$ \\
\hline Multiple-veteran household & & $0.802^{*}$ & $0.673,0.957$ & & 1.005 & $0.771,1.309$ \\
\hline \multicolumn{7}{|l|}{ Labour force status§ } \\
\hline \multicolumn{7}{|l|}{ Employed } \\
\hline Unemployed & & $2 \cdot 087^{\star \star \star}$ & $1.981,2.199$ & & $2 \cdot 138^{\star \star \star}$ & $2.002,2.284$ \\
\hline Out of the labour force & & 0.989 & $0.953,1.027$ & & 1.044 & $0.987,1.104$ \\
\hline Disabled & & $2 \cdot 116^{\star \star \star}$ & $2 \cdot 021,2 \cdot 216$ & & $2 \cdot 147^{\star \star \star}$ & $2.023,2.277$ \\
\hline Active military (non-veterans only) & & 0.782 & $0.610,1.002$ & & 0.715 & $0.457,1.120$ \\
\hline Female§ & & $1 \cdot 143^{\star \star \star}$ & $1.111,1.176$ & & $1.051^{*}$ & $1.008,1.097$ \\
\hline Age & & $1.064^{\star * *}$ & $1.059,1.070$ & & $1.092^{\star \star *}$ & $1.083,1.101$ \\
\hline Age-squared & & $0.999^{\star \star *}$ & $0.999,0.999$ & & $0.999^{\star \star \star}$ & $0.999,0.999$ \\
\hline \multicolumn{7}{|l|}{ Race/ethnicity§ } \\
\hline \multicolumn{7}{|l|}{ White, not Hispanic $\ddagger$} \\
\hline Black, not Hispanic & & $1.370^{\star \star \star}$ & $1.316,1.426$ & & $1.149^{\star \star \star}$ & $1.086,1.216$ \\
\hline Hispanic, any race & & $1 \cdot 224^{\star \star *}$ & $1.168,1.283$ & & 0.989 & $0.923,1.060$ \\
\hline American Indian, not Hispanic & & $1 \cdot 174^{\star}$ & $1.028,1.341$ & & $1.227^{\star}$ & $1.028,1.465$ \\
\hline Asian/Pacific Islander, not Hispanic & & $0.786^{\star \star *}$ & $0.720,0.857$ & & $0.631^{\star \star \star}$ & $0.544,0.732$ \\
\hline Multiple race, not Hispanic & & $1.543^{\star \star \star}$ & $1.397,1.705$ & & $1 \cdot 613^{\star \star \star}$ & $1.417,1.835$ \\
\hline \multicolumn{7}{|l|}{ Martial status§ } \\
\hline \multicolumn{7}{|l|}{ Married, spouse presentł } \\
\hline Married, spouse absent & & $1.393^{\star * *}$ & $1.269,1.529$ & & $1.537^{\star \star \star}$ & $1.351,1.748$ \\
\hline Widowed & & $1.423^{\star \star \star}$ & $1.343,1.507$ & & $1.591^{\star \star \star}$ & $1.462,1.731$ \\
\hline Divorced & & $1.637^{\star * *}$ & $1.574,1.702$ & & $1.863^{\star \star \star}$ & $1.760,1.971$ \\
\hline Separated & & $1.576^{\star \star \star}$ & $1.474,1.685$ & & $1.796^{\star \star \star}$ & $1.644,1.962$ \\
\hline Never married & & $1 \cdot 231^{\star \star \star}$ & $1.182,1.283$ & & $1 \cdot 338^{\star \star \star}$ & $1.261,1.421$ \\
\hline \multicolumn{7}{|l|}{ Maximum household education } \\
\hline \multicolumn{7}{|l|}{ Less than high schoolf } \\
\hline High school & & $0.861^{\star \star *}$ & $0.822,0.901$ & & $0.927^{*}$ & $0.872,0.985$ \\
\hline Some college & & $0.874^{\star \star \star}$ & $0.834,0.916$ & & 0.994 & $0.933,1.059$ \\
\hline Bachelor's degree & & $0.525^{\star \star \star}$ & $0.496,0.555$ & & $0.582^{\star \star \star}$ & $0.536,0.633$ \\
\hline Graduate school or higher & & $0.327^{\star \star \star}$ & $0.305,0.351$ & & $0.384^{\star \star *}$ & $0.343,0.430$ \\
\hline Number of children & & $0.962^{\star \star *}$ & $0.947,0.977$ & & $0.825^{\star \star *}$ & $0.804,0.846$ \\
\hline Number of adults & & $1.084^{\star \star *}$ & $1.066,1.103$ & & 1.023 & $0.997,1.049$ \\
\hline \multicolumn{7}{|l|}{ Housing type } \\
\hline \multicolumn{7}{|l|}{ House/apartment/flat $\ddagger$} \\
\hline Hotel or motel & & 1.208 & $0.875,1.666$ & & $1.501^{*}$ & $1.028,2 \cdot 191$ \\
\hline Mobile home & & $1.419^{\star \star \star}$ & $1.350,1.491$ & & $1 \cdot 337^{\star \star \star}$ & $1.250,1.430$ \\
\hline Other & & 0.736 & $0.505,1.074$ & & $1 \cdot 159$ & $0.742,1.810$ \\
\hline Housing tenure & & & & & & \\
\hline Owned or being purchased $\ddagger$ & & & & & & \\
\hline Rented for cash & & $1.682^{\star \star \star}$ & $1.631,1.734$ & & $1.776^{\star \star \star}$ & $1.696,1.859$ \\
\hline Occupied without payment & & $1 \cdot 317^{\star \star \star}$ & $1.197,1.448$ & & $1.483^{\star \star \star}$ & $1.307,1.682$ \\
\hline Immigrant household & & 1.020 & $0.976,1.066$ & & 0.943 & $0.882,1.007$ \\
\hline Household in poverty & & & & & & \\
\hline Noł & & & & & & \\
\hline Yes & & $2 \cdot 454^{\star \star \star}$ & $2.373,2.537$ & & $2 \cdot 678^{\star \star \star}$ & $2.539,2.826$ \\
\hline Not reported & & 1.049 & $0.991,1.109$ & & 1.096 & $0.999,1.203$ \\
\hline Receipt of food and nutrition assistance & & & & & & \\
\hline SNAP/Food Stamps participation & & $2 \cdot 526^{\star \star \star}$ & $2.427,2.628$ & & $2 \cdot 210^{\star \star \star}$ & $2.094,2.332$ \\
\hline WIC participation & & $1.095^{\star \star}$ & $1.026,1.169$ & & 0.935 & $0.859,1.018$ \\
\hline Free or reduced-price meals participation & & $2 \cdot 024^{\star \star \star}$ & $1.930,2 \cdot 124$ & & $1.567^{\star \star \star}$ & $1.466,1.675$ \\
\hline
\end{tabular}

SNAP, Supplemental Nutrition Assistance Program; WIC, Special Supplemental Nutrition Program for Women, Infants, and Children.

${ }^{\star} P<0.05,{ }^{\star \star} P<0.01,{ }^{* \star \star} P<0.001$.

†All models also control for respondent's state of residence and the year of data collection. All estimates are based on the use of sample weights provided by the Current Population Survey.

$\ddagger$ Referent category.

$\S$ Variable applies to the head of household in non-veteran homes or the veteran with the most recent service in veteran homes. 
Table 3 Unadjusted and adjusted past-year household food insecurity and very low food security by most recent period of military service among US households ( $n 388$ 680) from 2005-2013 waves of the Current Population Survey - Food Security Supplement†

\begin{tabular}{|c|c|c|c|c|c|c|}
\hline & \multicolumn{3}{|c|}{ Food insecurity } & \multicolumn{3}{|c|}{ Very low food security } \\
\hline & Probability & OR & $95 \% \mathrm{Cl}$ & Probability & OR & $95 \% \mathrm{Cl}$ \\
\hline \multicolumn{7}{|l|}{ Unadjusted results } \\
\hline \multicolumn{7}{|l|}{ Most recent period of military service } \\
\hline None (non-veteran)‡ & 0.144 & & & 0.054 & & \\
\hline September 2001 or later & 0.128 & $0.877^{\star *}$ & $0.795,0.969$ & 0.046 & $0.849^{*}$ & $0.724,0.995$ \\
\hline August 1990 to August 2001 & 0.131 & $0.895^{\star *}$ & $0.829,0.966$ & 0.049 & 0.896 & $0.795,1.010$ \\
\hline May 1975 to July 1990 & 0.131 & $0.895^{\star \star \star}$ & $0.842,0.952$ & 0.052 & 0.964 & $0.879,1.057$ \\
\hline Vietnam War (August 1964 to April 1975) & 0.075 & $0.483^{\star \star \star}$ & $0.455,0.513$ & 0.032 & $0.582^{\star \star *}$ & $0.531,0.637$ \\
\hline February 1955 to July 1964 & 0.046 & $0.290^{\star \star \star}$ & $0.259,0.324$ & 0.017 & $0.303^{\star \star \star}$ & $0.250,0.367$ \\
\hline Korean War or earlier & 0.034 & $0.208^{\star \star *}$ & $0.186,0.232$ & 0.013 & $0.226^{\star \star *}$ & $0.189,0.270$ \\
\hline \multicolumn{7}{|l|}{ Adjusted results } \\
\hline \multicolumn{7}{|l|}{ Most recent period of military service } \\
\hline None (non-veteran)‡ & 0.133 & & & 0.051 & & \\
\hline September 2001 or later & 0.135 & 1.013 & $0.904,1.136$ & 0.053 & 1.054 & $0.888,1.252$ \\
\hline August 1990 to August 2001 & 0.148 & $1 \cdot 172^{\star \star \star}$ & $1.072,1.280$ & 0.056 & $1 \cdot 133$ & $0.992,1.293$ \\
\hline May 1975 to July 1990 & 0.141 & $1.091^{*}$ & $1.015,1.171$ & 0.051 & 1.011 & $0.912,1.120$ \\
\hline Vietnam War (August 1964 to April 1975) & 0.125 & $0.912^{\star \star}$ & $0.852,0.976$ & 0.048 & 0.941 & $0.851,1.041$ \\
\hline February 1955 to July 1964 & 0.123 & 0.889 & $0.790,1.001$ & 0.045 & 0.873 & $0.715,1.065$ \\
\hline Korean War or earlier & 0.129 & 0.952 & $0.842,1.076$ & 0.054 & 1.082 & $0.891,1.316$ \\
\hline Multiple-veteran household & & $0.761^{\star *}$ & $0.636,0.912$ & & 0.964 & $0.738,1.261$ \\
\hline \multirow{2}{*}{\multicolumn{7}{|c|}{$\begin{array}{l}\text { Labour force status } \\
\text { Employed }\end{array}$}} \\
\hline & & & & & & \\
\hline Unemployed & & $2.089^{\star \star \star}$ & $1.983,2.201$ & & $2 \cdot 140^{\star * *}$ & $2.003,2.286$ \\
\hline Out of the labour force & & 0.993 & $0.957,1.032$ & & 1.046 & $0.989,1.107$ \\
\hline Disabled & & $2 \cdot 123^{\star \star \star}$ & $2.027,2.223$ & & $2 \cdot 151^{\star \star *}$ & $2 \cdot 027,2 \cdot 282$ \\
\hline Active military (non-veterans) & & 0.786 & $0.613,1.007$ & & 0.718 & $0.459,1.125$ \\
\hline Female§ & & $1 \cdot 139^{\star \star \star}$ & $1.107,1.173$ & & $1.050^{\star}$ & $1.007,1.095$ \\
\hline Age & & $1.063^{\star \star \star}$ & $1.057,1.069$ & & $1.092^{\star \star \star}$ & $1.083,1.101$ \\
\hline Age-squared & & $0.999^{\star \star \star}$ & $0.999,0.999$ & & $0.999^{\star \star \star}$ & $0.999,0.999$ \\
\hline \multirow{2}{*}{\multicolumn{7}{|c|}{$\begin{array}{l}\text { Race/ethnicity§ } \\
\text { White, not Hispanicł }\end{array}$}} \\
\hline & & & & & & \\
\hline Black, not Hispanic & & $1.366^{\star \star \star}$ & $1.312,1.423$ & & $1 \cdot 148^{\star \star \star}$ & $1.085,1.214$ \\
\hline Hispanic, any race & & $1.225^{\star \star \star}$ & $1.168,1.284$ & & 0.989 & $0.923,1.059$ \\
\hline American Indian, not Hispanic & & $1 \cdot 173^{\star}$ & $1.027,1.339$ & & $1.227^{*}$ & $1.028,1.464$ \\
\hline Asian/Pacific Islander, not Hispanic & & $0.785^{\star \star \star}$ & $0.720,0.856$ & & $0.630^{\star \star \star}$ & $0.543,0.732$ \\
\hline Multiple race, not Hispanic & & $1.538^{\star \star \star}$ & $1.392,1.699$ & & $1.610^{\star \star \star}$ & $1.415,1.832$ \\
\hline \multicolumn{7}{|l|}{ Martial status§ } \\
\hline \multicolumn{7}{|l|}{ Married, spouse presentł } \\
\hline Married, spouse absent & & $1.391^{\star * *}$ & $1.267,1.526$ & & $1.534^{\star \star *}$ & $1.349,1.745$ \\
\hline Widowed & & $1.400^{\star \star \star}$ & $1.320,1.485$ & & $1.579^{\star \star \star}$ & $1.450,1.721$ \\
\hline Divorced & & $1.632^{\star \star \star}$ & $1.569,1.697$ & & $1.859^{\star \star *}$ & $1.756,1.968$ \\
\hline Separated & & $1.573^{\star \star \star}$ & $1.471,1.681$ & & $1.793^{\star \star *}$ & $1.641,1.959$ \\
\hline Never married & & $1 \cdot 231^{\star \star \star}$ & $1 \cdot 182,1.283$ & & $1.339^{\star \star *}$ & $1.261,1.422$ \\
\hline \multirow{2}{*}{\multicolumn{7}{|c|}{$\begin{array}{l}\text { Maximum household education } \\
\text { Less than high schoolf }\end{array}$}} \\
\hline & & & & & & \\
\hline High school & & $0.862^{\star \star \star}$ & $0.824,0.903$ & & $0.928^{*}$ & $0.873,0.987$ \\
\hline Some college & & $0.875^{\star \star \star}$ & $0.835,0.917$ & & 0.994 & $0.933,1.060$ \\
\hline Bachelor's degree & & $0.525^{\star \star \star}$ & $0.497,0.556$ & & $0.582^{\star \star \star}$ & $0.536,0.633$ \\
\hline Graduate school or higher & & $0.327^{\star \star \star}$ & $0.305,0.352$ & & $0.384^{\star * *}$ & $0.344,0.430$ \\
\hline Number of children & & $0.961^{\star \star \star}$ & $0.947,0.976$ & & $0.824^{\star \star \star}$ & $0.804,0.845$ \\
\hline Number of adults & & $1.084^{\star \star *}$ & $1.065,1.102$ & & 1.022 & $0.997,1.048$ \\
\hline Housing type & & & & & & \\
\hline House/apartment/flatł & & & & & & \\
\hline Hotel or motel & & 1.207 & $0.874,1.665$ & & $1.502^{\star}$ & $1.029,2.192$ \\
\hline Mobile home & & $1.420^{\star \star \star}$ & $1.351,1.492$ & & $1.338^{\star \star \star}$ & $1.251,1.431$ \\
\hline Other & & 0.736 & $0.505,1.074$ & & $1 \cdot 160$ & $0.741,1.815$ \\
\hline Housing tenure & & & & & & \\
\hline Owned or being purchased $\ddagger$ & & & & & & \\
\hline Rented for cash & & $1.682^{\star \star \star}$ & $1.631,1.734$ & & $1.776^{\star \star \star}$ & $1.696,1.859$ \\
\hline Occupied without payment & & $1.315^{\star \star \star}$ & $1.196,1.447$ & & $1.483^{\star \star \star}$ & $1.307,1.682$ \\
\hline Immigrant household & & 1.019 & $0.976,1.065$ & & 0.943 & $0.882,1.007$ \\
\hline Household in poverty & & & & & & \\
\hline Noł & & & & & & \\
\hline Yes & & $2 \cdot 454^{\star \star \star}$ & $2 \cdot 374,2.537$ & & $2 \cdot 679^{\star \star \star}$ & $2 \cdot 539,2 \cdot 826$ \\
\hline Not reported & & 1.049 & $0.992,1.110$ & & 1.097 & $0.999,1.204$ \\
\hline Receipt of food and nutrition assistance & & & & & & \\
\hline SNAP/Food Stamps participation & & $2 \cdot 523^{\star \star \star}$ & $2.425,2.625$ & & $2 \cdot 209^{\star \star *}$ & $2 \cdot 093,2.331$ \\
\hline WIC participation & & $1.098^{\star \star}$ & $1.029,1.172$ & & 0.937 & $0.861,1.021$ \\
\hline Free or reduced-price meals participation & & $2 \cdot 030^{\star \star \star}$ & $1.935,2.130$ & & $1.569^{\star \star \star}$ & $1.468,1.677$ \\
\hline
\end{tabular}

SNAP, Supplemental Nutrition Assistance Program; WIC, Special Supplemental Nutrition Program for Women, Infants, and Children.

${ }^{\star} P<0.05,{ }^{* \star} P<0.01,{ }^{* * \star} P<0.001$.

†All models also control for respondent's state of residence and the year of data collection. All estimates are based on the use of sample weights provided by the Current Population Survey.

†Referent category.

$\S$ Variable applies to the head of household in non-veteran homes or the veteran with the most recent service in veteran homes. 
compared with $0 \cdot 144$ in non-veteran homes. Results were similar for VLFS: veteran households had significantly lower odds (OR $=0.601, P<0.001)$ of VLFS and the corresponding probability was substantially lower in veteran households (0.033 compared with 0.054). The bottom part of Table 2 presents estimates from fully controlled models. In these models, veteran status was no longer significantly associated with FI or VLFS.

In the top part of Table 3, which presents estimates from unadjusted models, the odds of FI were significantly lower for veterans of every period, especially those households with veterans of the Vietnam War era and earlier. Corresponding probabilities of FI were $50 \%$ or less (between 0.034 and 0.075) of those for non-veteran homes $(0 \cdot 144)$. The unadjusted results for VLFS showed a similar pattern, although the odds of VLFS were not statistically different for households with veterans from the August 1990 to August 2001 and May 1975 to July 1990 eras.

In results from adjusted models, which are reported in the lower part of Table 3, the findings were substantially changed: the odds of FI were not significantly different for household with veterans of the post 9/11 period, the period from 1955 to 1964 , and from the Korean War or earlier. Odds of FI for households with veterans from the Vietnam War era were still lower but with much smaller predicted differences in the probability of FI: $0.125 \mathrm{com}-$ pared with 0.133 for non-veteran households. Importantly, there was a change in direction of effect in adjusted models for households with veterans from the August 1990 to August 2001 and May 1975 to July 1990 eras, who had significantly higher odds $(\mathrm{OR}=1.172, \quad P=0.0005$ and $\mathrm{OR}=1 \cdot 091, P=0.0174)$ and probabilities $(0 \cdot 148$ and $0 \cdot 141)$ of FI. In the fully adjusted results, veteran status was not significantly associated with VLFS.

\section{Discussion}

The present study used nationally representative data on American households from 2005 to 2013 to estimate rates of FI in veteran and non-veteran households. In contrast to the limited body of previous research, our analyses found rates of FI (8.4\%) and VLFS (3.3\%) that were significantly and substantially lower in veteran households than in nonveteran households. In analyses that separated veteran households by their most recent period of service, rates of FI and VLFS were again significantly lower for households with veterans of nearly every group. Thus, our uncontrolled estimates based on national household data provide a very different picture from that suggested in previous research ${ }^{(1,2)}$, with most veteran households at significantly lower risk for FI and VLFS than non-veteran homes. We consider the principal value of these findings as contributing to a clearer picture of the basic prevalence of FI and VLFS among households with veterans, particularly given their stark contrast with previous research. However, we are careful not to over-emphasize these results, which do not account for the many differences between veteran and non-veteran households.

Rather, our adjusted models (which controlled for sociodemographic factors along with a number of additional factors that might reflect benefits that accrue to veteran households) are likely more informative for policy makers. In these models, the difference in predicted probability of FI between veteran and non-veteran households was very small and not statistically significant. Thus, differences in the distribution of characteristics among veteran and non-veteran households along with state and year controls explained the apparent average advantage that veteran households had regarding food security. However, controlled models that separated veteran households by period of most recent military service found that results did not generalize across veteran eras. In these models, households with veterans from the Vietnam War still had significantly lower predicted probability of FI $(0 \cdot 125)$, although the difference between the corresponding probability for non-veteran households $(0 \cdot 133)$ was much smaller than in uncontrolled estimates. Also, for households with veterans who served from 1990 to 2001 and from 1975 to 1990 , FI was significantly higher, $14.8 \%$ and $14 \cdot 1 \%$ respectively. In fact, results suggest a substantial change in FI between older veterans who served in the Vietnam War or earlier and recent veterans from 1975 onwards. In supplemental controlled analyses (available upon request), we combined veteran households into these two larger groups and found that the odds of FI were significantly higher (OR $=1.099, P=0.0006)$ among recent veterans and significantly lower $(\mathrm{OR}=$ $0.914, P=0.0025)$ among older veterans compared with non-veteran homes.

There are several possibilities underlying these results. First, this split between older and more recent veterans may reflect differences in the composition of the US Armed Forces coinciding with the onset of an all-volunteer force. Thus, the differences in FI may reflect these compositional differences such as higher proportions of volunteers from impoverished communities, families with dysfunction $^{(42,43)}$ and greater numbers of women. Further, the group of oldest veterans have accumulated a lifetime of advantages from the GI Bill and mortgage and healthcare programmes. More recent veteran cohorts have accrued these benefits for a shorter period of time. An alternative possibility is that the welfare of veteran groups has been determined in part by macro trends in the US economy. Whereas the oldest group of veterans returned to job opportunities and an expanding middle class, contemporary veterans have returned to stagnant wages and diminished wealth available to the middle class ${ }^{(44)}$. Newer veterans may also differ in the length and number of combat rotations, age at first entry into active duty and other factors. While our analyses controlled for a number of factors (household poverty, educational attainment and 
housing status) which would capture negative or positive selectivity, there may be subtle differences and interactions with the larger economy that we do not account for. Future research should seek to better understand the differences between veteran and non-veteran households and among veteran households, and how these differences contribute to material hardship like FI.

Perhaps most important, our results point to diversity among veteran households; one key finding is that households with veterans from the period May 1975 to August 2001 had slightly higher odds of FI than non-veteran households and post 9/11 veterans had no difference in odds. These results and those of the supplemental analyses described above suggest that recent veterans who served in 1975 or later may be at higher risk for FI and should be the recipients of targeted outreach to improve nutritional outcomes. We cannot project from these findings whether this disadvantage will continue, resolve or grow, but the food security of newgeneration veterans should be closely monitored. It is important to note that the higher odds of FI among some recent veterans were independent of participation in SNAP, WIC and the National School Lunch and School Breakfast Programs, which are the three largest nutrition programmes operated by the US Department of Agriculture. It may thus be necessary to explore whether different types of intervention or special outreach to optimize participation in these national programmes can reduce hardship. However, we also note that despite our use of controlled models, our results do not allow us to make causal inferences regarding veteran status and food insecurity, so additional research is necessary before making firm policy recommendations.

The present study was not without limitations. The CPS contained only limited information on the experiences of veterans. The survey lacked information about length of service, deployment, combat exposure, rank and branch, and the nature of separation or discharge from the armed forces, all of which might help to better distinguish among different groups of veterans. The CPS is also crosssectional, which precluded analyses that might have described the dynamic nature of FI in households. Lastly, because it is a household survey, the CPS does not include information on homeless veterans, who are inevitably at much higher risk of FI than veterans with more stable housing arrangements. Nationally representative data suggest that despite significant decreases in the past five years, there are still nearly 50000 homeless veterans and veterans are over-represented in the homeless population in the $\mathrm{USA}^{(45,46)}$. Accordingly, the present findings are best categorized as representative of the differences in FI among veteran and non-veteran households. Additional analyses are necessary to understand whether accounting for homelessness among both veterans and non-veterans would affect the nature of the findings reported here.

\section{Conclusion}

In conclusion, using a large, nationally representative sample of all American households, these analyses represent an important complement to previous research that is based on highly selected groups of veterans or used a more limited measure of FI. Other study strengths are the use of nutrition programme participation and detailed demographic variables to explore many of the differences between veteran and non-veteran households that are likely linked to FI. While on balance veteran households were at substantially lower risk of FI than comparable civilian households, there is reason for concern among the most recent veterans. These findings support continued monitoring of the well-being of recent veterans and exploration of targeted outreach to ensure their full participation in nutrition programmes and other benefits.

\section{Acknowledgements}

Financial support: This research received no specific grant from any funding agency in the public, commercial or not-for-profit sectors. Conflicts of interest: None. Authorship: D.P.M. helped conceptualize the study, prepared and analysed the data, and led in the writing of the manuscript. M.J.L. helped to conceptualize the study, assisted in the interpretation of results and assisted in the writing of the manuscript. T.B. assisted in the interpretation of results and assisted in the writing of the manuscript. E.D. assisted in the interpretation of results and assisted in the writing of the manuscript. Ethics of buman subject participation: All analyses were conducted with de-identified secondary data with no means to link information to individual respondents; therefore the study was considered to be not human subjects research and required no review by an institutional review board.

\section{Supplementary material}

To view supplementary material for this article, please visit http://dx.doi.org/10.1017/S1368980015003067

\section{References}

1. Widome R, Jensen A, Bangerter A et al. (2015) Food insecurity among veterans of the US wars in Iraq and Afghanistan. Public Health Nutr 18, 844-849.

2. Wang EA, McGinnis KA, Goulet J et al. (2015) Food insecurity and health: data from the Veterans Aging Cohort Study. Public Health Rep 130, 261-268.

3. Feeding America (2014) Hunger in America 2014: Military service in Feeding America client households, background and calculations. http://help.feedingamerica. org/HungerInAmerica/HIA-2014-Military.pdf (accessed May 2015). 
4. Weinfeld NS, Mills G, Borger C et al. (2015) Hunger in America 2014 - National Report Prepared for Feeding America. Chicago, IL: Feeding America; available at http://help.feedingamerica.org/HungerInAmerica/hunger-inamerica-2014-full-report.pdf

5. Coleman-Jensen A, Gregory C \& Singh A (2014) Housebold Food Security in the United States in 2013. Economic Research Report no. ERR-173. Washington, DC: US Department of Agriculture, Economic Research Service; available at http://www.ers.usda.gov/publications/erreconomic-research-report/err173.aspx

6. Cook JT, Frank DA, Berkowitz C et al. (2004) Food insecurity is associated with adverse health outcomes among human infants and toddlers. J Nutr 134, 1432-1438.

7. Stuff JE, Casey PH, Szeto KL et al. (2004) Household food insecurity is associated with adult health status. J Nutr 134, $2330-2335$.

8. Hampton T (2007) Food insecurity harms health, well-being of millions in the United States. JAMA 298, 1851-1853.

9. Rose D (1999) Economic determinants and dietary consequences of food insecurity in the United States. J Nutr 129, 2 S Suppl., 517S-520S.

10. Noonan K, Corman H \& Reichman NE (2014) Effects of maternal depression on family food insecurity. http://www. ukcpr.org/sites/ukcpr.org/files/documents/DP2014-07_0.pdf (accessed May 2015).

11. Larson MJ, Adams RS, Mohr BA et al. (2013) Rationale and methods of the Substance Use and Psychological Injury Combat Study (SUPIC): a longitudinal study of Army service members returning from deployment in FY2008-2011. Subst Use Misuse 48, 863-879.

12. Seal KH, Bertenthal D, Miner CR et al. (2007) Bringing the war back home: mental health disorders among 103,788 US veterans returning from Iraq and Afghanistan seen at Department of Veterans Affairs facilities. Arch Intern Med 167, 476-482.

13. Mansfield AJ, Bender RH, Hourani LL et al. (2011) Suicidal or self-harming ideation in military personnel transitioning to civilian life. Suicide Life Threat Behav $\mathbf{4 1}$, 392-405.

14. Friedman MJ, Schnurr PP \& McDonagh-Coyle A (1994) Post-traumatic stress disorder in the military veteran. Psychiatr Clin North Am 17, 265-278.

15. Bohnert AS, Ilgen MA, Bossarte RM et al. (2012) Veteran status and alcohol use in men in the United States. Mil Med 177, 198-203.

16. Capone C, McGrath AC, Reddy MK et al. (2013) Traumarelated correlates of alcohol use in recently deployed OEF/ OIF veterans. J Trauma Stress 26, 354-360.

17. Hoge CW, Auchterlonie JL \& Milliken CS (2006) Mental health problems, use of mental health services, and attrition from military service after returning from deployment to Iraq or Afghanistan. JAMA 295, 1023-1032.

18. Milliken CS, Auchterlonie JL \& Hoge CW (2007) Longitudinal assessment of mental health problems among active and reserve component soldiers returning from the Iraq war. JAMA 298, 2141-2148.

19. Seal KH, Cohen G, Waldrop A et al. (2011) Substance use disorders in Iraq and Afghanistan veterans in VA healthcare, 2001-2010: implications for screening, diagnosis and treatment. Drug Alcohol Depend 116, 93-101.

20. Kleykamp M (2013) Unemployment, earnings and enrollment among post 9/11 veterans. Soc Sci Res $\mathbf{4 2}$, 836-851.

21. Humensky JL, Jordan N, Stroupe KT et al. (2012) How are Iraq/Afghanistan-era veterans faring in the labor market? Armed Forces Soc 39, 158-183.

22. Institute of Medicine (2010) Returning home from Iraq and Afghanistan: preliminary assessment of readjustment needs of veterans, service members, and their families.
http://www.iom.edu/Reports/2010/Returning-Home-fromIraq-and-Afghanistan-Preliminary-Assessment.aspx (accessed May 2015).

23. Elbogen EB, Sullivan CP, Wolfe J et al. (2013) Homelessness and money mismanagement in Iraq and Afghanistan veterans. Am J Public Health 103, Suppl. 2, S248-S254.

24. National Center for Veterans Analysis and Statistics (n.d.) Profile of veterans: 2012 data from the American Community Survey. http://www.va.gov/vetdata/docs/Special Reports/Profile_of_Veterans_2012.pdf (accessed May 2015).

25. Kramarow EA \& Pastor PN (2012) The health of male veterans and nonveterans aged 25-64: United States, 20072010. NCHS Data Brief no. 101. http://www.cdc.gov/nchs/ data/databriefs/db101.pdf (accessed May 2015).

26. US Department of Veterans Affairs (2015) Veterans Benefits Administration. http://www.benefits.va.gov/benefits/ (accessed May 2015).

27. US Department of Veterans Affairs (2015) Homeless programs. http://www.va.gov/homeless (accessed May 2015).

28. National Center for Veterans Analysis and Statistics (2015) Veteran's Supplemental Nutritional Assistance Program (SNAP) participants: 2013 American Community Survey. http://www.va.gov/vetdata/docs/SpecialReports/SNAPVe terans2013.pdf (accessed May 2015).

29. Gilroy CL, Phillips RL \& Blair JD (1990) The all-volunteer army: fifteen years later. Armed Forces Soc 16, 329-350.

30. Cooper RV (1977) Military Manpower and the all-Volunteer Force. Santa Monica, CA: Rand.

31. Polich JM (1981) Epidemiology of alcohol abuse in military and civilian populations. Am J Public Health 71, 1125-1132.

32. Rosenheck R, Frisman L \& Chung AM (1994) The proportion of veterans among homeless men. Am J Public Health 84 , 466-469.

33. Greenberg GA, Rosenheck RA \& Desai RA (2007) Risk of incarceration among male veterans and nonveterans: are veterans of the all volunteer force at greater risk? Armed Forces Soc 33, 337-350.

34. Fargo J, Metraux S, Byrne T et al. (2012) Prevalence and risk of homelessness among US veterans. Prev Chronic Dis 9 , E45.

35. Greenberg GA \& Rosenheck RA (2007) Are male veterans at greater risk for nonemployment than nonveterans? Monthly Lab Rev 130, 23-31.

36. Heflin CM, Wilmoth JM \& London AS (2012) Veteran status and material hardship: the moderating influence of work-limiting disability. Soc Serv Rev 86, 119-142.

37. Wilmoth JM, London AS \& Heflin CM (2015) Economic well-being among older-adult households: variation by veteran and disability status. J Gerontol Soc Work 58, 399-419.

38. Coleman-Jensen A, Nord M \& Singh AS (2013) Household Food Security in the United States in 2012. Economic Research Report no. ERR-155. Washington, DC: US Department of Agriculture, Economic Research Service; available at http://www.ers.usda.gov/ersDownloadHandler. ashx?file $=/$ media $/ 1183208 /$ err-155.pdf

39. Coleman-Jensen A, Nord M, Andrews M et al. (2012) Household Food Security in the United States in 2011. Economic Research Report no. ERR-141. Washington, DC: US Department of Agriculture, Economic Research Service; available at http://www.ers.usda.gov/media/884525/err141. pdf

40. Bickel G, Nord M, Price C et al. (2000) Guide to Measuring Household Food Security - Revised 2000. Alexandria, VA: US Department of Agriculture, Food and Nutrition Service; available at http://www.fns.usda.gov/guide-measuringhousehold-food-security-revised-2000

41. Nord M \& Bickel G (2002) Measuring Children's Food Security in US Households, 1995-1999. Food Assistance and Nutrition Research Report no. 25. Washington, DC: US Department of Agriculture, Economic Research 
Service; available at http://www.ers.usda.gov/media/889965/ fanrr25_002.pdf

42. Larson MJ, Wooten NR, Adams RS et al. (2012) Military combat deployments and substance use: review and future directions. J Soc Work Pract Addict 12, 6-27.

43. Kelley ML, Runnals J, Pearson MR et al. (2013) Alcohol use and trauma exposure among male and female veterans before, during, and after military service. Drug Alcohol Depend 133, 615-624.

44. Faberman RJ \& Foster T (2013) Unemployment among recent veterans during the Great Recession. Econ Perspect 37, 1-13.
45. Henry M, Cortes A, Shivji A et al. (2014) The 2014 Annual Homelessness Assessment Report (AHAR) to Congress: Part 1, Point-in-Time Estimates of Homelessness. Washington, DC: US Department of Housing and Urban Development, Office of Community Planning and Development; available at https://www.hudexchange.info/resources/ documents/2014-AHAR-Part1.pdf

46. National Center for Veterans Analysis and Statistics (2012) Profile of sheltered homeless veterans for fiscal years 2009 and 2010. http://www.va.gov/vetdata/docs/SpecialReports/ Homeless_Veterans_2009-2010.pdf (accessed May 2015). 